\title{
Aerobic Fitness Level Affects Cardiovascular and Salivary Alpha Amylase Responses to Acute Psychosocial Stress
}

Thomas Wyss ${ }^{1 *}$, Maria Boesch ${ }^{2,3}$, Lilian Roos ${ }^{1}$, Céline Tschopp ${ }^{1}$, Klaus M. Frei ${ }^{1}$, Hubert Annen ${ }^{3}$ and Roberto La Marca²

\begin{abstract}
Background: Good physical fitness seems to help the individual to buffer the potential harmful impact of psychosocial stress on somatic and mental health. The aim of the present study is to investigate the role of physical fitness levels on the autonomic nervous system (ANS; i.e. heart rate and salivary alpha amylase) responses to acute psychosocial stress, while controlling for established factors influencing individual stress reactions.

Methods: The Trier Social Stress Test for Groups (TSST-G) was executed with 302 male recruits during their first week of Swiss Army basic training. Heart rate was measured continuously, and salivary alpha amylase was measured twice, before and after the stress intervention. In the same week, all volunteers participated in a physical fitness test and they responded to questionnaires on lifestyle factors and personal traits. A multiple linear regression analysis was conducted to determine ANS responses to acute psychosocial stress from physical fitness test performances, controlling for personal traits, behavioural factors, and socioeconomic data.

Results: Multiple linear regression revealed three variables predicting $15 \%$ of the variance in heart rate response (area under the individual heart rate response curve during TSST-G) and four variables predicting $12 \%$ of the variance in salivary alpha amylase response (salivary alpha amylase level immediately after the TSST-G) to acute psychosocial stress. A strong performance at the progressive endurance run (high maximal oxygen consumption) was a significant predictor of ANS response in both models: low area under the heart rate response curve during TSST-G as well as low salivary alpha amylase level after TSST-G. Further, high muscle power, non-smoking, high extraversion, and low agreeableness were predictors of a favourable ANS response in either one of the two dependent variables.
\end{abstract}

Conclusions: Good physical fitness, especially good aerobic endurance capacity, is an important protective factor against health-threatening reactions to acute psychosocial stress.

Keywords: Physical fitness, Physical activity, Stress response, Stress prevention, Autonomic nervous system, Cross-stressor adaptation hypotheses

Abbreviations: ANS, Autonomic nervous system; AUCg, Area under the curve; BMT, Basic military training; HPA, Hypothalamic-pituitary-adrenal axis; HR, Heart rate; IPAQ, International Physical Activity Questionnaire; OLS, Oneleg standing test; PER, Progressive endurance run; PFC, Prefrontal cortex; PSQ, Perceived stress questionnaire; RS, Resilience scale; SAA, Salivary alpha amylase; SL, Standing long jump; SSP, Seated shot put; TMS, Trunk muscle strength test; TSST-G, Trier Social Stress Test for Groups; $\dot{V O}_{2}$ max, Maximal oxygen consumption; $\triangle \mathrm{SAA}$, Change in salivary alpha amylase

\footnotetext{
* Correspondence: Thomas.wyss@baspo.admin.ch

${ }^{1}$ Swiss Federal Institute of Sport Magglingen (SFISM), Magglingen, Switzerland
}

Full list of author information is available at the end of the article 


\section{Key Points}

1. Aerobic fitness level affects cardiovascular responses to acute psychosocial stress.

2. Aerobic fitness level affects alpha amylase responses to acute psychosocial stress.

3. Present results support the cross-stressor adaptation hypothesis, saying that even if psychological coping with stress remains unaffected, the various traininginduced adaptations in the organization of the ANS of an aerobically fit person influences physiological responses to acute stress.

\section{Background}

Psychosocial stress has a harmful impact on somatic and mental health $[1,2]$. In order to improve individual resistance to psychosocial stress, regular physical activity seems to be an effective strategy [3-7]. This relation is based on similarities between physiological processes during physical and psychological stress $[8,9]$. A situation which is perceived as stressful, independent of whether the source is physical $[10,11]$ or psychological $[12,13]$, leads to an altered activity of the hypothalamicpituitary-adrenal (HPA) axis and the autonomic nervous system (ANS). Because regular physical training has been shown to diminish the reactivity of the HPA axis and ANS during exercises [10,11], a cross-adaptation to psychological stress is assumed [14]. To test the crossstressor adaptation hypothesis [15] discussed above, many studies investigated the relation between physical activity behaviour and psychological stress reactivity. However, the health-promoting effect of regular physical exercise on stress reactivity is most probably based on a combination of two effects: better psychological resources resulting in improved psychological coping with stress and (2) reduced physiological reactivity to stress [14]. De Geus and Stubbe believe that even if psychological coping with stress remains unaffected, the various training-induced adaptations in the organization of the ANS and its target organs influence the pattern and intensity of physiological responses to stress [14]. To investigate that hypothesis, the influence of physical fitness on stress responses should be investigated while controlling for physical activity behaviour and potential stress-influencing factors. As potential stress-influencing factors, personality- and behaviour-related factors were only selected when prior studies have demonstrated their relation to stress reactions. Some evidence suggests that personality traits, resilience, smoking habits, educational level, and migrating background are influencing perception and responses to psychosocial stress [16-20].

It is the aim of the present study to investigate the role of physical fitness level on ANS (i.e. heart rate and salivary alpha amylase) responses to acute psychosocial stress, while controlling for established factors influencing individual stress reactions. Using the framework of the cross-stressor adaptation in the present study, the hypothesis was that the individual physical fitness level modifies the physiological stress responses to non-exercise stressors. Lower baseline heart rate (HR), stronger HR reaction to non-exercise stressors, faster HR recovery after termination of a non-exercise stressor, and lower salivary alpha amylase (sAA) immediately after the non-exercise stressor are expected in physically welltrained subjects.

\section{Methods}

\section{Study Design and Participants}

Volunteers were recruited among the 693 young men who started their mandatory military service in the Swiss Armed Forces garrison located in Aarau, Switzerland. This sample is representative of the healthy young Swiss male population. In total, 651 recruits $(94 \%)$ gave their informed consent to volunteer in the scientific project, approved by the local Ethics Committee of the Canton Aargau, Switzerland (registration number 2011/008). All research was performed in accordance with the ethical standards of the Declaration of Helsinki. For the present study, 302 recruits were randomly selected from the pool of volunteers to take part in a psychosocial stress examination. In the first week of basic military training (BMT), the selected volunteers participated in an acute psychosocial stress test (Trier Social Stress Test for Groups [TSST-G]), while HR was monitored continuously and saliva samples were collected immediately before and after the TSST-G for analyses of sAA. In the same week, all volunteers participated in a physical fitness test and they responded to a battery of questionnaires regarding lifestyle factors and personal traits.

\section{Stress Provocation}

To induce acute psychosocial stress, the TSST-G was applied as stated by Bösch et al. [21] and first described by von Dawans et al. [22]. The TSST-G is an established, standardized performance test protocol that combines a high level of social-evaluative threat and uncontrollability to provoke psychosocial stress [21]. In the present study, four participants were seated next to each other but separated by privacy protection walls. After $2 \mathrm{~min}$ of baseline measurement, the upcoming task was introduced and subjects were allowed to prepare themselves for $2 \mathrm{~min}$. Then, a fake expert panel entered the room and turned on video cameras to make the subjects believe that they were being videotaped. Each participant had $2 \mathrm{~min}$ to introduce himself for a mock job interview. After completion of the four short interviews, participants were asked to perform a mental arithmetic task consisting of continuous subtraction, as quickly and accurate as possible. After each 
mistake, subjects had to restart from the beginning. Again, this examination took $2 \mathrm{~min}$ for each of the four participants. Finally, the TSST-G was followed by $6 \mathrm{~min}$ of recovery measurement.

\section{Data Collection}

Heart rate was continuously measured during the stress examination using an ambulatory electrocardiography system (Equivital System; Hidalgo, Cambridge, UK). Data were edited manually (VivoSense, Vivonoetics, San Diego, CA), and the average HR (in beats per minute [bpm]) was calculated for 2-min time intervals.

Salivary alpha amylase samples were collected immediately before and after the TSST-G. Participants were requested to gently chew on a Salivette (Sarstedt, Sevelen, Switzerland) for $1 \mathrm{~min}$. The samples were stored at $-20^{\circ} \mathrm{C}$ until analyses were conducted in the biochemical laboratory of the Department of Clinical Psychology and Psychotherapy at the University of Zurich, Switzerland. The activity of sAA was analysed with a kinetic colorimetric test using assay kits from Roche (Roche 11555685 alpha-Amylase Liquid acc, Roche, Basel, Switzerland) and an automatic analyser (Biotek Instruments, Lucern, Switzerland) with the software KC4 (Roche).

Physical fitness was assessed using the Swiss Army physical fitness test battery [23]. The test battery contained a progressive endurance run (PER) to measure aerobic endurance capacity; a standing long jump (SLJ) and a seated shot put (SSP) to measure muscle power of the lower and upper extremities, respectively; a trunk muscle strength test (TMS) to measure trunk muscle fitness; and a one-leg standing test (OLS) to measure balance. The PER is a paced running test, conducted on a 400-m outdoor track according to the protocol developed by Conconi et al. [24] and evaluated using the final running velocity. Based on PER, the maximal oxygen consumption $\left(\dot{\mathrm{V}}_{2} \max \right)$ can be estimated using the formula by Wyss et al. [23]. The SLJ was performed from the gym hall floor onto a mat of 7-cm height. The SSP was performed as a 2-kg ball chest pass while sitting upright on a bench with the back touching a solid wall. In the TMS, the subjects had to hold an isometric body position (prone bridge) [25] for as long as possible, while lifting their feet alternately. In OLS, participants had to keep position on one leg as long as possible while closing their eyes after $10 \mathrm{~s}$ and laying their head back while keeping the eyes closed after $20 \mathrm{~s}$. Time was measured for the left and right legs separately, and the sum of both was used as a value for balance ability. Precise descriptions of the five tests were published elsewhere [23]. To assess anthropometry, body height was measured to the nearest $0.1 \mathrm{~cm}$ using a stadiometer (Seca model 214, Seca GmbH, Hamburg, Germany) and body weight to the nearest $0.1 \mathrm{~kg}$ on a calibrated digital balance (Seca model 877, Seca GmbH, Hamburg, Germany).

Data on personality traits, behavioural factors, and socioeconomic data were assessed by questionnaires. Data on the personality of participants were assessed using the German version of the NEO Psychological Personality Inventory with 240 items and five-point Likert scale by Ostendorf and Angleitner [26]. Participants' resilience was assessed by the short version of the resilience scale (RS) [27], consisting of 11 items answered on a seven-point Likert scale. The German version of the perceived stress questionnaire (PSQ) [28] with 20 items and a four-point Likert scale was used to detect subjects' perceived chronic stress level. Physical activity behaviour was assessed using the International Physical Activity Questionnaire (IPAQ short) [29], and participants were classified as inactive, partly active, unregularly active, regularly active, and trained. Further, questions that were previously not validated about smoking habits, migration background, and educational level were used to group participants as smoker and non-smoker and Swiss citizens with and without a migration background and in three categories of educational levels (lower secondary school, upper secondary school, and high school).

\section{Statistical Analysis}

Statistical analyses were performed using SPSS for Windows (version 22.0, IBM, Chicago, IL) with a level of significance of $p<.05$ to indicate statistical significance. Group results are presented as mean \pm standard deviation. A multiple linear regression was run to predict ANS responses to acute psychosocial stress from physical fitness test performances, controlling for personal traits, behavioural factors, and socioeconomic data. As a dependent variable representing magnitude of cardiovascular response, the area under the individual HR response curve (AUCg) was calculated using a trapezoid formula [30], as has been done before to represent physiological responses [5]. As a dependent variable representing sympathetic sAA reactivity, the increase from pre- to post-TSST-G as well as the sAA level determined immediately after the TSST-G (i.e. post level) was used. The latter variable was included because baseline levels usually constitute a period of combined sympathetic and, probably more important, parasympathetic activity, and the latter can positively influence amylase activity itself $[31,32]$. Therefore, it is unclear which of both variables, the change value or the value determined after the stress test, better reflects sympathetic stress reactivity. The potential independent predicting variables are listed in Table 1. Regression analyses were performed twofold, with the same result: by backwards elimination and by systematic entering of the variables in the order of the strength of the association with the dependent 
Table 1 Potential predicting variables for ANS stress reactivity

\begin{tabular}{|c|c|}
\hline Physical fitness & Volunteers' mean values \\
\hline $\begin{array}{l}\text { Aerobic fitness by progressive } \\
\text { endurance run }\left(\mathrm{V}_{2} \mathrm{max}\right)\end{array}$ & $50.1 \pm 5.0 \mathrm{ml} / \mathrm{kg} / \mathrm{min}$ \\
\hline $\begin{array}{l}\text { Muscle power of the lower } \\
\text { extremities by standing long jump }\end{array}$ & $2.3 \pm 0.2 \mathrm{~m}$ \\
\hline $\begin{array}{l}\text { Muscle power of the upper } \\
\text { extremities by seated shot put }\end{array}$ & $6.4 \pm 0.7 \mathrm{~m}$ \\
\hline $\begin{array}{l}\text { Trunk muscle fitness by trunk } \\
\text { muscle strength test }\end{array}$ & $133.3 \pm 64.0 \mathrm{~s}$ \\
\hline $\begin{array}{l}\text { Balance by one-leg } \\
\text { standing test }\end{array}$ & $47.1 \pm 9.9 \mathrm{~s}$ \\
\hline $\begin{array}{l}\text { Anthropometry by body } \\
\text { mass index }\end{array}$ & $23.6 \pm 3.0 \mathrm{~kg} / \mathrm{m}^{2}$ \\
\hline Covariates & $\begin{array}{l}\text { Volunteers' mean } \\
\text { values or proportion }\end{array}$ \\
\hline $\begin{array}{l}\text { Physical activity behaviour } \\
\text { by IPAQ short (index) }\end{array}$ & $\begin{array}{l}59.4 \% \text { regularly } \\
\text { active or trained }\end{array}$ \\
\hline \multicolumn{2}{|l|}{ Personality by NEO } \\
\hline Neuroticism (index 0-192) & $76 \pm 19$ \\
\hline Extraversion (index 0-192) & $118 \pm 20$ \\
\hline $\begin{array}{l}\text { Openness for experiences } \\
\text { (index 0-192) }\end{array}$ & $108 \pm 16$ \\
\hline Agreeableness (index 0-192) & $108 \pm 16$ \\
\hline Conscientiousness (index 0-192) & $117 \pm 20$ \\
\hline Perceived stress by PSQ (index 0-1) & $0.3 \pm 0.2$ \\
\hline Resilience by RS short (index 0-77) & $60 \pm 10$ \\
\hline Smoking status (yes/no) & $34.0 \%$ yes \\
\hline Migration background (yes/no) & $18.9 \%$ yes \\
\hline Educational level (low, medium, high) & $\begin{array}{l}31.3 \% \text { low, } 41.8 \% \\
\text { medium, } 26.9 \% \text { high }\end{array}$ \\
\hline
\end{tabular}

ANS autonomic nervous system

variable. Variables which did not predict the dependent variable significantly $(p>.05)$ were removed from the model. The assumptions of linearity, non-collinearity, independence of errors, homoscedasticity, unusual points, and normality of residuals were met. The effect size of predicting variables was calculated according to Cohen [33] as $f^{2}=\frac{\left(R_{\text {including }}^{2}-R_{\text {excluding }}^{2}\right)}{1-R_{\text {including }}^{2}}$. Cohen termed an effect size of $.02, .15$, and .35 as small, medium, and large, respectively. Fitness variables remaining in the final regression model were used to stratify volunteers in four performance groups of a similar number of participants for each performance test. For example, the $25 \%$ of participants with the best PER performances were stratified to the first quartile, representing volunteers with highest aerobic fitness level, while the fourth quartile represented those $25 \%$ with the lowest aerobic fitness level. HR reactivity was calculated by the absolute difference in HR values in the 2-min segment before the TSST-G and the 2-min segment of the interview task during the TSST-G (first HR-peak). HR recovery was calculated by the absolute difference in HR values in the 2-min segment of the mental arithmetic task (second HRpeak) and the 2-min segment starting 6 min after the end of the TSST-G. Group comparisons were conducted using independent sample $t$ tests. Furthermore, repeated measures ANOVA was used to investigate effects of group, time, and group $\times$ time interaction on HR and sAA levels during TSST-G. In all the presented data, the Mauchly test showed that the assumption of sphericity has been violated; therefore, the Greenhouse-Geisser estimate was used to report repeated measures ANOVA results.

\section{Results}

\section{Participants}

Complete data on 219 male recruits $(73 \%$ of the total sample of selected volunteers) with a mean age of $20.2 \pm$ 1.1 years, a body size of $177.8 \pm 6.6 \mathrm{~cm}$, and a body mass of $74.9 \pm 11.3 \mathrm{~kg}$ were registered. Volunteers' mean values and proportions for all independent variables are presented in Table 1. Participants' $\mathrm{V}_{2}$ max was significantly correlated to the SLJ, and SSP performance $(r=.190$ and -.190 , $p<.01$, respectively), while the two latter ones were positively correlated to each other as well $(r=.180, p=.007)$. Further, participants' $\dot{\mathrm{V}}_{2}$ max was significantly correlated to their physical activity and smoking behaviour as well as to their educational level $(r=.163,-.186$, and .170, $p<.05$, respectively), and their smoking habit was correlated with their educational level and agreeableness $(r=.347$, and $.236, p<.001$, respectively). Participants that were classified as insufficiently physically active (less than $150 \mathrm{~min}$ of moderate or $75 \mathrm{~min}$ of vigorous physical activities per week) had a lower $\dot{\mathrm{VO}}_{2}$ max level, compared to the participants who were sufficiently physically active $(48.87 \pm 4.46$ and $50.99 \pm 5.10 \mathrm{ml} / \mathrm{kg} / \mathrm{min}, p=.002$ ).

\section{Cardiac Response to Acute Stress}

A multiple linear regression predicting HR-AUCg revealed performances at the PER, at the SLJ, and smoking attitude as significant independent variables (Table 2). The many other covariates-BMI, physical activity behaviour, resilience, personality traits, educational level, and migration background-proved statistically insignificant. The assumptions of linearity, non-collinearity, independence of errors, homoscedasticity, unusual points, and normality of residuals were met by the used model (Table 2). The variables in the final regression model significantly predicted HR-AUCg, $F=12.626, p<.001$, adj. $R^{2}=.150$. Regression coefficients, kept on measurement scale, standard errors, and their respective $95 \%$ confidence intervals (CIs) can be derived from Table 2. 
Table 2 Summary of multiple regression analysis of HR-AUCg

\begin{tabular}{llllllll}
\hline Variable & $B$ & SE & $\beta$ & $t$ & Sig & Cl (95) \\
\hline Intercept & 1573.776 & 257.158 & & 6.120 & 0.000 & 1066.903 & 2080.649 \\
Progressive endurance run (s) & -0.447 & 0.087 & -0.336 & -5.145 & 0.000 & -0.618 & -0.276 \\
Standing long jump (m) & 420.851 & 112.601 & 0.240 & 3.738 & 0.000 & 198.908 & 642.793 \\
Smoking (0=no; 1 =yes) & -114.471 & 40.811 & -0.180 & -2.805 & 0.005 & -194.913 & -34.030 \\
\hline
\end{tabular}

HR-AUC $g$ area under the HR response curve, $B$ unstandardized regression coefficient, SE standard error of the coefficient, $\beta$ standardized coefficient, Sig significance, $\mathrm{Cl}$ (95) $95 \%$ confidence interval

${ }^{\text {a }}$ Smoking status before military service

The strongest influence on total magnitude of HR response to acute stress was found for aerobic fitness (Cohen's effect size of $f^{2}=.12$ ). Participants were, therefore, stratified into four groups of aerobic fitness, with 55 volunteers in the first quartile of aerobic fitness $\left(\dot{\mathrm{VO}}_{2} \mathrm{max}\right.$ of $\left.55.8 \pm 1.6 \mathrm{ml} / \mathrm{kg} / \mathrm{min}\right)$ and 54 volunteers in the fourth quartile $\left(\mathrm{V}_{2} \max\right.$ of $\left.43.3 \pm 2.6 \mathrm{ml} / \mathrm{kg} / \mathrm{min}\right)$. The HR responses to the TSST-G in these groups are presented in Fig. 1. As Fig. 1 indicates, a repeated measures ANOVA showed a significant main effect on HR by the factors group ( $F=10.622, p=.002)$ and time $(F=19.271, p<.001)$, but not by the interaction between group and time $(F=1.618, p=.137)$. HR values were significantly different $(p<.05)$ between groups at baseline. However, HR reactivity in participants of high aerobic fitness level was stronger $(+11.9 \pm 10.1 \mathrm{bpm})$ than that in participants of low aerobic fitness level $(+6.4 \pm 9.1 \mathrm{bpm}$, $p=.004)$. HR recovery was not different between the two groups $(-11.3 \pm 10.8$ and $-9.4 \pm 7.2$ for first and fourth quartiles, respectively, $p=.370$ ).

The same comparison, using SLJ performance to stratify volunteers in different groups, resulted in Fig. 2. The 52 volunteers in the first quartile of the lower extremity muscle power had a better SLJ performance $(2.5 \pm 0.1 \mathrm{~m})$ than that of the 56 volunteers in the fourth quartile (2.1 $\pm .1 \mathrm{~m})$. The HR response to the TSST-G in those two SLJ performance groups is presented in Fig. 2. As Fig. 2 indicates, a repeated measures ANOVA showed a significant main effect on HR by the factors group $(F=4.469$, $p=.037)$ and time $(F=24.560, p<.001)$ as well as the interaction between group and time $(F=3.259, p=.003)$. $\mathrm{HR}$ values of the two performance groups were not different before and after the TSST-G intervention. However, HR reactivity in the first quartile of SLJ performances was stronger $(+13.5 \pm 13.3 \mathrm{bpm})$ than that in participants of the fourth quartile of SLJ performances $(+4.8 \pm 9.8 \mathrm{bpm}, p=.001)$. HR recovery was not different between the two groups $(-12.0 \pm 9.4$ and $-9.8 \pm 8.2$ for the first and fourth quartiles, respectively, $p=.221$ ).

Smokers and non-smokers had the same baseline and recovery HR before and after the TSST-G. However, non-smokers showed a stronger HR reaction to the TSST-G $(+10.5 \pm 9.7 \mathrm{bpm})$ compared to smokers $(+6.0 \pm 7.6, p=.001)$.

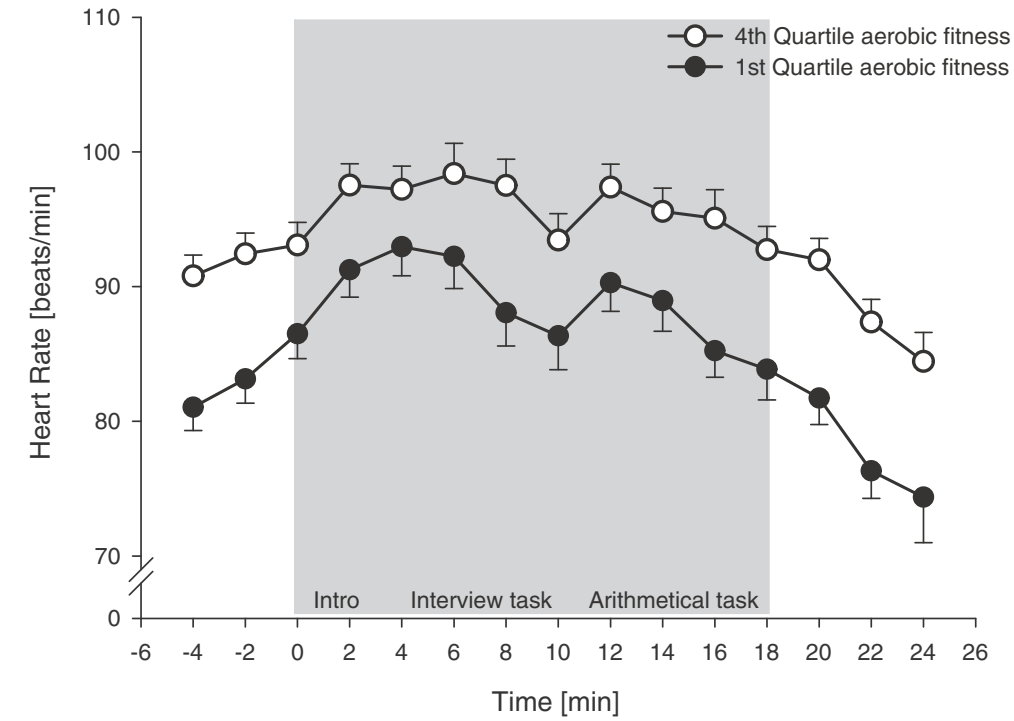

Fig. $1 \mathrm{HR}$ before, during, and after the TSST-G among volunteers of high and low aerobic fitness. Values are presented as mean \pm SEM for each 2-min time interval. The first quartile represents subjects of high aerobic fitness; the fourth quartile represents subjects of low aerobic fitness. Each of the 15 time-segments in the figure represents data from all 219 subjects. HR heart rate, TSST-G Trier Social Stress Test for Groups 


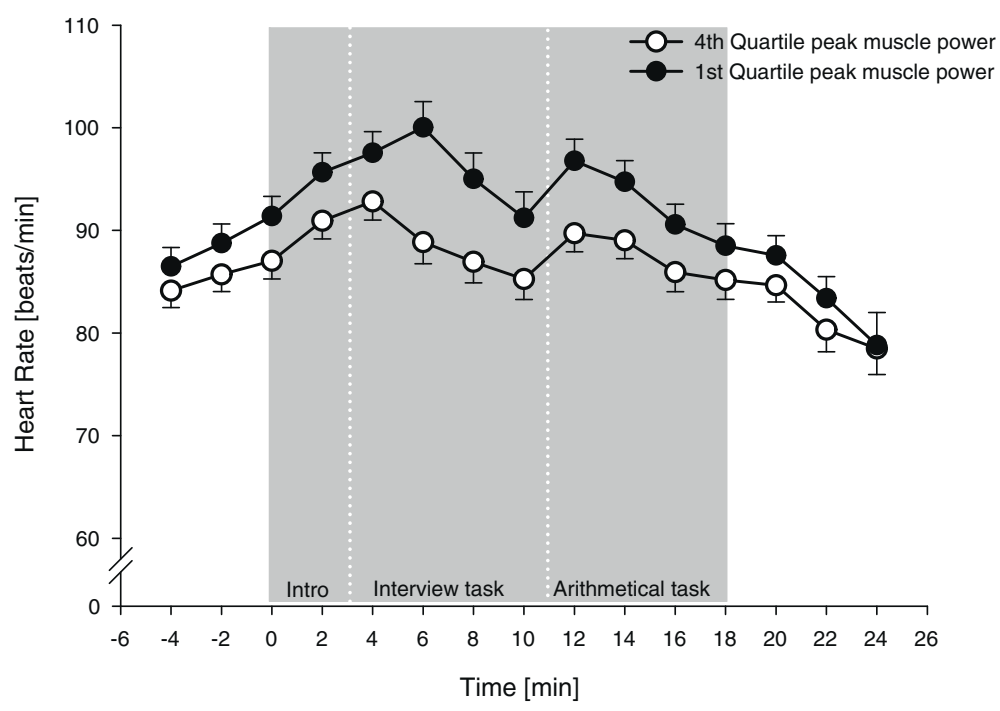

Fig. 2 HR before, during, and after the TSST-G among volunteers of high and low muscle power in the lower extremities. Values are presented as mean \pm SEM for each 2-min time interval. The first quartile represents subjects of high muscle power. The fourth quartile represents subjects of low muscle power. Each of the 15 time-segments in the figure represents data from all 219 subjects. HR heart rate, TSST-G Trier Social Stress Test for Groups

Subjects' HR-peak levels during the interview task (first HR-peak) and the mental arithmetic task (second HRpeak) were comparable $(93.2 \pm 16.4$ and $92.2 \pm 14.6 \mathrm{bpm}$, $p=.166)$. However, HR during those 2-min time intervals were higher $(p<.001)$ than the task-specific average HR over $8 \mathrm{~min}(91.6 \pm 14.4 \mathrm{bpm}$ during interview task and $89.7 \pm 13.2$ bpm during arithmetic task).

\section{sAA Response to Acute Stress}

A multiple linear regression predicting the change in salivary alpha amylase $(\triangle \mathrm{sAA})$ values (increase in sAA level from pre- to post-TSST-G) revealed performance at the OLS $(B=.764, \mathrm{SE}=.313, \beta=.170, t=2.437, p$ $=.016)$ and educational level $(B=13.079, \mathrm{SE}=4.127, \beta$ $=.223, t=3.169, p=.002)$ as the only significant independent variables. The variables in the final regression model explained $5 \%$ of the variance in the $\triangle \mathrm{sAA}$ values, $F=2.825, p=.012$, and adj. $R^{2}=.050$.

A multiple linear regression predicting the sAA stress response to acute stress (sAA level after TSST-G) revealed performance at the PER, performance at the OLS, and the two personality traits "extraversion" as well as "agreeableness" as relevant independent variables (Table 3). The variables in the final regression model significantly predicted the sAA stress response, $F=8.796, p<.001$, and adj. $R^{2}=.124$. Regression coefficients, kept on measurement scale, standard errors, and their respective $95 \%$ CIs can be derived from Table 3 .

Aerobic fitness was the only variable represented in the models, predicting HR and SAA responses to acute stress. Cohen's effect size for PER as a predictor variable for the sAA stress response was $f^{2}=.03$. The sAA values immediately before and after the TSST-G of recruits in the quartile of the lowest and highest aerobic fitness level are presented in Fig. 3. A repeated measures ANOVA with time (before and after TSST-G) as withinsubjects factor and group affiliation as between-subjects factor showed a significant main effect on sAA values by the factors group $(F=10.369, p=.002)$ and time $(F=$ $16.434, p<.001)$, but not for the interaction between group and time $(F=0.474, p=.492)$.

\section{Discussion}

HR and sAA responses to acute psychosocial stress were shown to be dependent on recruits' physical fitness level, even when controlled for physical activity behaviour and further relevant covariates. Therefore, the present study supports the hypothesis of De Geus and Stubbe [14] that the various fitness-related adaptations in the organization of the ANS and its target organs influence the pattern and intensity of physiological responses to stress, even if psychological coping with stress remains unaffected. HRAUCg was predicted by performances at the PER, SLJ, and regular smoking behaviour (Table 2). sAA stress responses were predicted by performance at the PER and OLS, as well as by values representing the personality trait "extraversion" and "agreeableness" (Table 3). Surprisingly, physical activity behaviour did not remain in the regression models, and only a weak relation to aerobic fitness was demonstrated $(r=.163)$. That might be due to the methodological disadvantages of self-reported questionnaires to assess physical activity [34]. In the following sections, the relationship of each predicting variable to 
Table 3 Summary of multiple regression analysis of the sAA stress response

\begin{tabular}{llllllll}
\hline Variable & $B$ & SE & $\beta$ & $t$ & Sig & Cl (95) \\
\hline Intercept & -23.049 & 35.859 & & 0.644 & 0.520 & -47.579 & 93.778 \\
Progressive endurance run (s) & -0.048 & 0.017 & -0.182 & -2.821 & 0.005 & -0.081 & -0.014 \\
One-leg standing test (s) & 1.538 & 0.393 & 0.249 & 3.909 & 0.000 & 0.762 & 2.313 \\
Extraversion (index) & -0.496 & 0.197 & -0.163 & -2.524 & 0.012 & -0.884 & -0.109 \\
Agreeableness (index) & 0.702 & 0.228 & 0.200 & 3.080 & 0.002 & 0.253 & 1.150
\end{tabular}

SAA salivary alpha amylase, $B$ unstandardized regression coefficient, SE standard error of the coefficient, $\beta$ standardized coefficient, Sig significance, $C I$ (95) $95 \%$ confidence interval

HR-AUCg and SAA is discussed and compared to results of previous studies.

\section{Cardiac Response to Acute Stress}

The cumulative HR-AUCg value, as an index of the total HR output across the measurement period, has been shown to be helpful to detect relevant variables, predicting cardiac response to acute stress [5]. However, HRAUCg is strongly dependent on three aspects of HR development, on baseline $\mathrm{HR}, \mathrm{HR}$ reactivity during TSST-G, and on HR recovery after TSST-G. A healthy HR reaction to stress can be described as low baseline $\mathrm{HR}$, strong $\mathrm{HR}$ reaction to acute stress, and fast $\mathrm{HR}$ recovery after the acute stress situation [35]. A low baseline or resting heart rate was found in the review of Cooney et al. [36] to be strongly related to reduced incidence of cardiovascular diseases. A meta-analysis about the relation between cardiovascular responses to acute stress and health revealed that a greater reactivity to stress was associated with poor cardiovascular health status [37]. However, included studies usually considered changes in systolic and diastolic blood pressure as a marker for cardiovascular responses. Studies examining HR reaction to acute stress, instead, showed higher increases in HR during acute stress in healthy subjects. For example, in the study of Jackson et al. [13], subjects with lower risk of hypertension showed a HR increase of $20 \mathrm{bpm}$ during mental arithmetic task compared to their

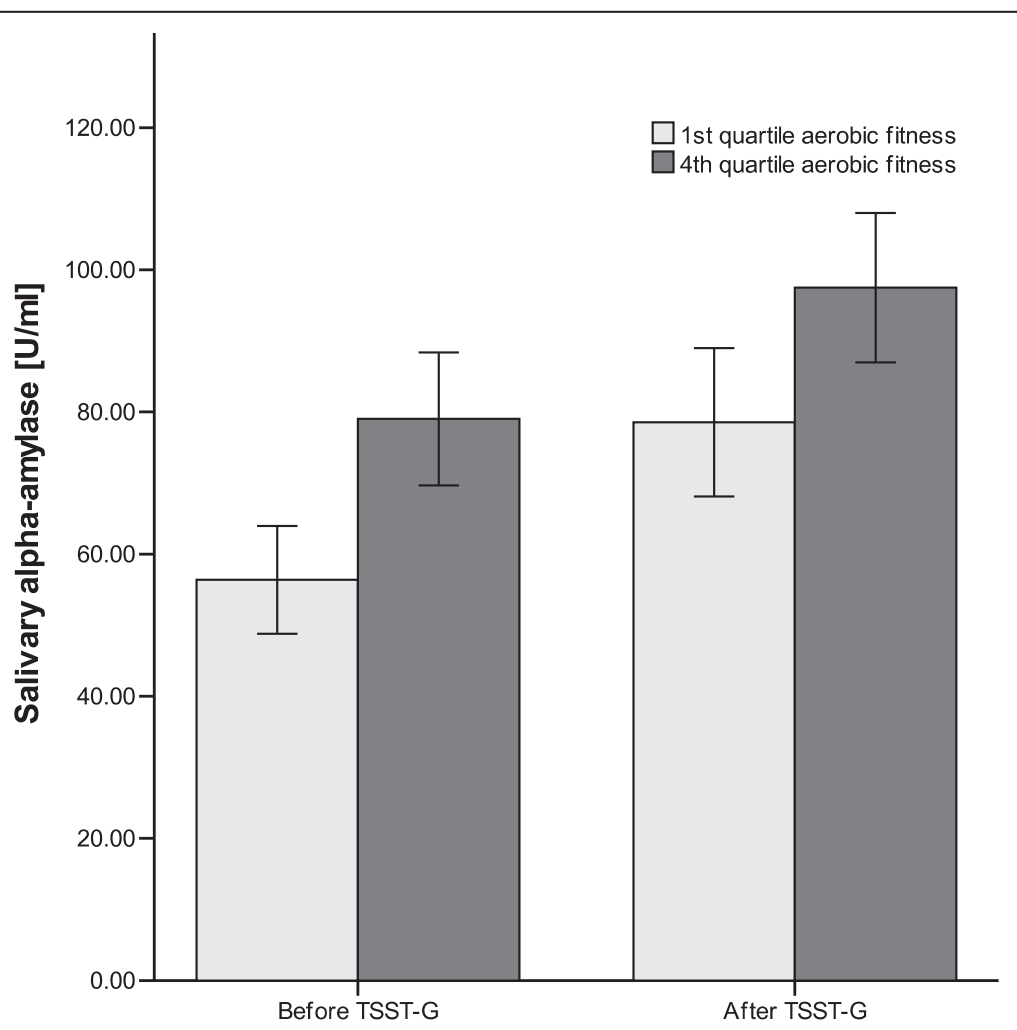

Fig. 3 Salivary alpha amylase stress response in subjects of high and low aerobic fitness. Values are presented as mean \pm SEM. Salivary alpha amylase was investigated immediately before and after the Trier Social Stress Test for Groups (TSST-G). The first quartile represents subjects of high aerobic fitness. The fourth quartile represents subjects of low aerobic fitness 
peers with higher risk of hypertension with a $\mathrm{HR}$ increase of $12 \mathrm{bpm}$. This observation can be explained by the evolutionary importance to get ready to fight or flight as soon as a threat appears. Furthermore, with regard to the law of initial values (assuming that the magnitude of responses is dependent of the initial baseline level, i.e. a high baseline is associated with a reduced reactivity), one could expect a higher HR reactivity in subjects with lower baseline levels [38]. Nevertheless, further evidence arose showing a decreased HR reaction to acute stress to be related with worse health $[35,39,40]$. Therefore, one has to assume that both exaggerated as well as blunted cardiovascular reactivity can be maladaptive $[41,42]$. Finally, a fast HR recovery from acute stress was consistently associated with reduced cardiovascular risk status [37].

A good aerobic endurance capacity is related to healthy cardiac reaction to acute stress. Two out of three criteria of a healthy HR reaction on stress were demonstrated in volunteers in the first quartile of PER performance. They showed a baseline HR of $10 \mathrm{bpm}$ lower than that of volunteers in the fourth quartile of PER. Further, they revealed a significantly greater HR reactivity to acute stress compared to the lowest quartile of cardiovascular fitness. A meta-regression analysis of 73 studies investigating laboratory stress came to the same conclusion that cardiorespiratory fitness is related to greater reactivity and better recovery of HR responses [43]. In contrast, other meta-analyses found a decreased psychological stress reactivity in relation with physical fitness (e.g. Forcier et al. [44]), contributing to the inconsistency in the literature.

Based on the present data, muscle power might be related to a healthy cardiac reaction to acute stress as well (strong HR reactivity and fast HR recovery). Subjects of low and high SLJ performance showed the same baseline HR before the stress task. However, during the TSSTG, the group of subjects with high SLJ performance showed a stronger HR reaction, and a trend of faster HR recovery (Fig. 2), resulting in larger HR-AUCg values. It can be concluded that subjects with high muscle power showed two out of three criteria of a healthy reaction to stress (strong HR reactivity and fast HR recovery) [35] compared to subjects with low muscle power. Based on prior studies on stress perception, we conclude that higher muscle power might lead to healthier physiological reactions to stress as well as to lower perceived stress level as well [45-47]. However, to the knowledge of the authors, the relation between anaerobic fitness, or muscle strength, and physiological responses to non-training-related, acute psychosocial stress is not sufficiently investigated yet. Therefore, further studies in this field are necessary to discuss the results demonstrated in the present study.

Smoking is related to an unhealthy cardiac reaction to acute stress. In agreement with our results, Child and Wit
[48] demonstrated smaller relative HR reactivity during a TSST for smokers, compared to non-smokers. Therefore, the ability to adequately react to a stressor seems reduced in smokers. Authors of prior studies suggest that smoking causes a transient but also a long-term reduction in vagal cardiac control in young people $[48,49]$.

The many other covariates-BMI, physical activity behaviour, resilience, personality traits, educational level, and migration background-proved statistically insignificant in the multivariate regression analysis of the present study.

\section{sAA Response to Acute Stress}

Nater and Rohlender [50] demonstrated in their review that the saliva enzyme sAA is a sensitive biomarker for stress-related changes in the body that reflects the activity of the sympathetic nervous system. Therefore, they emphasized that sAA level is a good marker to represent stress reactions. Balodis et al. [51] demonstrated a significant positive relation between subjective stress perception (anxiety reactivity) and the increase in sAA from pre- to post-TSST-G as well as the level of sAA after the TSST-G. In conclusion, high $\triangle \mathrm{sAA}$ and high post-sAA values are related to increased perception of threat in a stressful situation.

A good aerobic endurance capacity might be positively related to healthy sAA reactivity on acute stress. A repeated measures ANOVA analysis demonstrated aerobic fitness-group affiliation to be a significant main effect on sAA, with low physical fitness related to increased sAA values. This result was previously observed in other studies and explained by the cross-stressor adaptation hypothesis. According to the cross-stressor adaptation hypothesis, in physically trained individuals, lower sAA responses to stressors other than the training exercise can be expected [15, 52].

In the present study, volunteers of high-balance ability (assessed by OLS) showed higher $\triangle$ sAA values over the acute stress period as well as higher sAA values after the TSST-G. No similar results of other studies exist. However, this positive relation was not expected and does not go in line with the results of Nedeljkovic et al. [53].

Educational level might be related to stress reactivity as well. However, the results of different studies are not consistent. In the present study, a positive relation between educational level and $\triangle \mathrm{sAA}$ response on acute stress was found. In accordance with our study, Fiocco et al. [54] found higher cortisol reactivity on acute stress in subjects with high educational level. In contrast to our results, a study on stress perception derived reduced perceived stress level in subjects of higher education [20].

Extraversion and agreeableness remained in the regression model, predicting sAA level after TSST-G. The present results indicate that high extraversion leads to reduced sAA reactivity, while high agreeableness leads 
to increased sAA values after the TSST-G. Therefore, high extraversion and low agreeableness are favourable personality traits in terms of favourable reaction to acute psychosocial stress. Prior studies showed the same relation between extraversion and perceived psychological stress $[16,55]$. However, these studies showed no significant relation between agreeableness and perceived psychological stress.

\section{Relation Between Physical Activity, Physical Fitness, and Stress Reactivity}

Regular participation in physical activity (e.g. exercise) is expected to influence strength, balance, and aerobic capacity, three aspects of physical fitness [34]. Exercise results in acute and chronic changes in central and autonomic nervous system activity, the contracting muscles, and the heart, with several mechanisms being involved, including the exercise pressor reflex, arterial and cardiopulmonary baroreceptors, and the central command (for an overview see Fisher et al. [56]). As addressed by the cross-stressor hypothesis [15], physical activity, exercise training, and physical fitness are related to increased parasympathetic and decreased sympathetic activity, myocardial hypertrophy, increased stroke volume, reduced blood pressure during rest, and an increase in muscle capillary density and force [57]. Additionally, beneficial effects were reported with regard to mental health (e.g. depression and anxiety disorders) [58], cognitive function, and the central nervous system, with greater white and grey matter volume [59-61], mainly in the hippocampus and prefrontal cortex (PFC) [34]. Furthermore, an inverse association with central [62] and cardiac oxidative stress [63] and inflammation [64] was found. All together, these findings support the health-protecting and health-promoting effect of physical activity.

When comparing brain and peripheral structures and mechanisms affected by exercise, a wide overlap with structures involved in stress reactivity becomes evident. In fact, the neurophysiological model introduced by Lovallo [42] assumes that differences in stress reactivity derive from differences in three organizational levels. Level I includes the prefrontal cortex (PFC) and the limbic system. Level II addresses the hypothalamus and the brain stem consisting of output signals to the body and feedback to level I. Level III includes the peripheral tissues, with difference reflecting among other things' variations in autonomic or endocrine output.

Therefore, exercise (or fitness), which is related to better mood and PFC characteristics, seems to show higher activity in level I, eliciting stronger inhibitory control over limbic structures crucial in the control of cardiac activity [65]. When confronted with stressors, the top-down modulation from the PFC to the central nucleus of the amygdala is disinhibited, resulting in multiple mechanisms being activated or inhibited (level II) leading to an increase in HR (level III). These mechanisms include direct activation as well as disinhibition of sympathoexcitatory neurons in the rostral ventrolateral medulla, and inhibition of the nucleus of the solitary tract with subsequent inhibition of vagal motor outputs in the nucleus ambiguous and dorsal vagal motor nucleus [65]. This results in an increase of sympathetic and a decrease in parasympathetic activity and, therefore, a rise in HR. In subjects with higher physical fitness, one can assume higher inhibitory control from the PFC and, therefore, higher parasympathetic and lower sympathetic activity during rest. Nevertheless, when stressed, a fitter subject has the potential to show a larger disinhibition compared to a less fit subject, since the latter probably experiences less inhibition by the PFC already under rest, limiting the capacity to further inhibit the activity of the limbic system. As a consequence of the larger disinhibition in fitter subjects, the net reduction in vagal and increase in sympathetic activity can be larger, allowing for a stronger $\mathrm{HR}$ reactivity to stress. These considerations might explain our findings of lower HR under rest and stronger HR stress reactivity in participants with strong aerobic fitness.

\section{Limitations}

In order to measure the genuine stress reactivity of recruits before the influence of any training, reactivity to the TSST-G had to be assessed during their first week of BMT. Since we had only 5 days to investigate volunteers' stress reactivity, it was not possible to perform the timeconsuming TSST-G with all the 651 volunteers. However, we were able to investigate 302 randomly chosen volunteers.

Due to organizational reasons, we were not able to provide $60 \mathrm{~min}$ to adapt participants to the laboratory setting, as recommended for the assessment of psychoneuroendocrine baseline data [51]. Unfortunately, due to the short adaptation period, some subjects probably did not reach a calm resting state of mind when sAA baseline data was assessed immediately before the TSST-G.

Salivary samples were only taken twice. More sAA samples before, during, and after the TSST-G would have been more meaningful. Possibly, the peak in sAA response was not assessed due to our study design. However, more salivary samples would have distracted participants from the situation, causing a psychosocial stressful situation.

The order of active participation of the four subjects during the mock job interview and mental arithmetical task was not recorded. In future studies, this information should be recorded in order to compare physiological reactions while actually performing the stress-inducing task and while passively attending and observing the others being stressed. 
The present study group does only represent young, healthy men. Our results cannot be generalized per se for women, older men, and physically or mentally ill populations.

\section{Conclusions}

The present study demonstrates that good physical fitness-especially, good aerobic endurance capacity-is an important protective factor against healththreatening reactions to acute psychosocial stress. Cardiovascular fitness remains a significant predictor variable, even when controlled for the most important influencing factors to individual stress reactions. These results have important implications for individuals but also organizations providing mentally and physically demanding jobs. Employers should support and offer access to physical endurance training for their staff, especially in settings where employees regularly have to cope with stressful situations. In conclusion, regular and good physical training of individuals and employees to increase their cardiovascular fitness is not only important for their physical health but also their ability to adequately react to acute psychosocial stress situations.

\section{Acknowledgements}

We would like to thank all volunteering recruits and their military commanders as well as the school commander of the Swiss Armed Forces training school Inf DD 142/143 of Kdo 14 located in Aarau, Switzerland, who agreed to participate in this time-consuming scientific project.

\section{Funding}

This study received funding from the Federal Department of Defence, Civil Protection and Sports DDPS, Switzerland.

\section{Authors' Contributions}

TW was involved in the conception, data collection, analysis, manuscript writing and revision. MB and LR performed the main part of the data collection and were involved in the data analysis and revision. CT prepared the theoretical background and was involved in editing the manuscript. KF performed the data analysis and revision. HA was involved in all aspects of this study, including conception, data collection, data analysis, and revision. RL was involved in the conception of the study, manuscript writing, and critical revision. All authors read and approved the final manuscript.

\section{Competing Interests}

The authors declare that they have no competing interests.

\section{Ethics Approval and Consent to Participate}

The present study was approved by the local Ethics Committee of the Canton Aargau, Switzerland (registration number 2011/008). All subjects gave their informed consent to volunteer in the scientific project. All research was performed in accordance with the ethical standards of the Declaration of Helsinki.

\section{Author details}

${ }^{1}$ Swiss Federal Institute of Sport Magglingen (SFISM), Magglingen, Switzerland. ${ }^{2}$ Clinical Psychology and Psychotherapy, University of Zurich, Zurich, Switzerland. ${ }^{3}$ Military Academy, Swiss Federal Institute of Technology, ETH Zurich, Zurich, Switzerland.

Received: 5 February 2016 Accepted: 3 August 2016

Published online: 23 August 2016

\section{References}

1. Ehlert U, Gaab J, Heinrichs M. Psychoneuroendocrinological contributions to the etiology of depression, posttraumatic stress disorder, and stress-related bodily disorders: the role of the hypothalamus-pituitary-adrenal axis. Biol Psychol. 2001;57(1-3):141-52. doi:S0301051101000928.

2. Vanitallie TB. Stress: a risk factor for serious illness. Metabolism. 2002;51 (6 Suppl 1):40-5. doi:ameta0510s40.

3. Dishman RK, Jackson EM, Nakamura Y. Influence of fitness and gender on blood pressure responses during active or passive stress. Psychophysiology. 2002;39(5):568-76. 10.1017.S0048577202394071.

4. Rimmele U, Zellweger BC, Marti B, Seiler R, Mohiyeddini C, Ehlert U, et al. Trained men show lower cortisol, heart rate and psychological responses to psychosocial stress compared with untrained men.

Psychoneuroendocrinology. 2007;32(6):627-35. doi:S0306-4530(07)00086-8.

5. Rimmele U, Seiler R, Marti B, Wirtz PH, Ehlert U, Heinrichs M. The level of physical activity affects adrenal and cardiovascular reactivity to psychosocial stress. Psychoneuroendocrinology. 2009;34(2):190-8. doi:10.1016/.jpsyneuen. 2008.08.023.

6. Dishman RK, Berthoud HR, Booth FW, Cotman CW, Edgerton VR, Fleshner MR, et al. Neurobiology of exercise. Obesity. 2006;14(3):345-56. doi:14/3/345.

7. Petruzzello SJ, Jones AC, Tate AK. Affective responses to acute exercise: a test of opponent-process theory. J Sports Med Phys Fitness. 1997;37(3):205-12.

8. Holmes DS, Roth DL. Association of aerobic fitness with pulse rate and subjective responses to psychological stress. Psychophysiology. 1985;22(5): 525-9.

9. Hull EM, Young SH, Ziegler MG. Aerobic fitness affects cardiovascular and catecholamine responses to stressors. Psychophysiology. 1984;21(3): 353-60.

10. Deuster PA, Chrousos GP, Luger A, DeBolt JE, Bernier LL, Trostmann UH, et al. Hormonal and metabolic responses of untrained, moderately trained, and highly trained men to three exercise intensities. Metabolism. 1989;38(2): 141-8. doi:0026-0495(89)90253-9.

11. Luger A, Deuster PA, Kyle SB, Gallucci WT, Montgomery LC, Gold PW, et al. Acute hypothalamic-pituitary-adrenal responses to the stress of treadmill exercise. Physiologic adaptations to physical training. N Engl J Med. 1987; 316(21):1309-15. doi:10.1056/NEJM198705213162105.

12. Moyna NM, Bodnar JD, Goldberg HR, Shurin MS, Robertson RJ, Rabin BS. Relation between aerobic fitness level and stress induced alterations in neuroendocrine and immune function. Int J Sports Med. 1999;20(2):136-41. doi:10.1055/s-2007-971107.

13. Jackson EM, Dishman RK. Hemodynamic responses to stress among black women: fitness and parental hypertension. Med Sci Sports Exerc. 2002;34(7): 1097-105.

14. de Geus EJ, Stubbe JH. Aerobic exercise and stress reduction. In: Fink G, editor. Encyclopedia of stress. New York: Academic; 2007. p. 73-8.

15. Sothmann MS, Buckworth J, Claytor RP, Cox RH, White-Welkley JE, Dishman RK. Exercise training and the cross-stressor adaptation hypothesis. Exerc Sport Sci Rev. 1996;24:267-87.

16. Penley JA, Tomaka J, Wiebe JS. The association of coping to physical and psychological health outcomes: a meta-analytic review. J Behav Med. 2002; 25(6):551-603

17. Southwick SM, Vythilingam M, Charney DS. The psychobiology of depression and resilience to stress: implications for prevention and treatment. Annu Rev Clin Psychol. 2005;1:255-91. doi:10.1146/annurev. clinpsy.1.102803.143948.

18. Parrott AC. Smoking cessation leads to reduced stress, but why? Int J Addict. 1995;30(11):1509-16.

19. Parrott AC. Cigarette smoking: effects upon self-rated stress and arousal over the day. Addict Behav. 1993;18(4):389-95. doi:03064603(93)90055-E.

20. Williams DR, Yan Y, Jackson JS, Anderson NB. Racial differences in physical and mental health: socio-economic status, stress and discrimination. J Health Psychol. 1997;2(3):335-51. doi:10.1177/135910539700200305.

21. Boesch M, Sefidan S, Ehlert U, Annen H, Wyss T, Steptoe A, et al. Mood and autonomic responses to repeated exposure to the Trier Social Stress Test for Groups (TSST-G). Psychoneuroendocrinology. 2014;43:41-51.

22. von Dawans B, Kirschbaum C, Heinrichs M. The Trier Social Stress Test for Groups (TSST-G): a new research tool for controlled simultaneous social stress exposure in a group format. Psychoneuroendocrinology. 2011;36:514-22. 
23. Wyss T, Marti B, Rossi S, Kohler U, Mäder U. Assembling and verification of a fitness test battery for the recruitment of the Swiss army and nation-wide use. Swiss J Sports Med Sports Traumat. 2007;55(4):126-31.

24. Conconi F, Ferrari M, Ziglio PG, Droghetti P, Codeca L. Determination of the anaerobic threshold by a noninvasive field test in runners. J Appl Physiol. 1982;52(4):869-73.

25. Wunderlin S, Roos L, Roth R, Faude O, Frey F, Wyss T. Trunk muscle strength tests to predict injuries, attrition and military ability in soldiers. J Sports Med Phys Fitness. 2015;55(5):535-43. doi:R40Y9999N00A150043.

26. Ostendorf F, Angleitner A. NEO-Persönlichkeitsinventar nach Costa und McCrae: Revidierte Fassung. Göttingen: Hogrefe; 2004.

27. Schumacher J, Leppert K, Gunzelmann T, Strauss B, Brähler E. Die Resilienzskala - Ein Fragebogen zur Erfassung der psychischen Widerstandsfähigkeit als Personmerkmal. Z Klin Psychol Psychiatr Psychother. 2005;53(1):16-39.

28. Fliege $H$, Rose M, Arck P, Walter OB, Kocalevent R-D, Weber C, et al. The perceived stress questionnaire (PSQ) reconsidered: validation and reference values from different clinical and healthy adult samples. Psychosom Med. 2005;67(1):78-88. doi:10.1097/01.psy.0000151491.80178.78.

29. Craig $C L$, Marshall AL, Sjostrom M, Bauman AE, Booth ML, Ainsworth BE, et al. International Physical Activity Questionnaire: 12-country reliability and validity. Med Sci Sports Exerc. 2003;35(8):1381-95. doi:10.1249/01.MSS. 0000078924.61453.FB.

30. Pruessner JC, Kirschbaum C, Meinlschmid G, Hellhammer DH. Two formulas for computation of the area under the curve represent measures of total hormone concentration versus time-dependent change. Psychoneuroendocrinology. 2003;28(7):916-31. doi:S0306453002001087.

31. Bosch JA, Veerman EC, de Geus EJ. a-Amylase as a reliable and convenient measure of sympathetic activity: don't start salivating just yet! Psychoneuroendocrinology. 2011;36(4):449-53. doi:10.1016/j. psyneuen.2010.12.019.

32. Busch L, Sterin-Borda L, Borda E. An overview of autonomic regulation of parotid gland activity: influence of orchiectomy. Cells Tissues Organs. 2006; 182(3-4):117-28

33. Cohen J. A power primer. Psychol Bull. 1992;112(1):155-9.

34. Erickson $\mathrm{Kl}$, Leckie RL, Weinstein AM. Physical activity, fitness, and gray matter volume. Neurobiol Aging. 2014;35 Suppl 2:S20-8.

35. Carroll D, Phillips AC, Hunt K, Der G. Symptoms of depression and cardiovascular reactions to acute psychological stress: evidence from a population study. Biol Psychol. 2007;75(1):68-74. doi:S0301-0511(06)00258-4.

36. Cooney MT, Vartiainen E, Laatikainen T, Juolevi A, Dudina A, Graham IM. Elevated resting heart rate is an independent risk factor for cardiovascular disease in healthy men and women. Am Heart J. 2010;159(4):612-9. doi:10. 1016/j.ahj.2009.12.029. e3.

37. Chida Y, Steptoe A. Greater cardiovascular responses to laboratory mental stress are associated with poor subsequent cardiovascular risk status: a meta-analysis of prospective evidence. Hypertension. 2010;55(4):1026-32. doi:10.1161/HYPERTENSIONAHA.109.146621.

38 Berntson GG, Uchion BN, Cacioppo JT. Origins of baseline variance and the law of initial values. Psychophysiology. 1994;31(2):204-10.

39 Kupper N, Denollet J, Widdershoven J, Kop WJ. Cardiovascular reactivity to mental stress and mortality in patients with heart failure. JACC Heart Fail. 2015;3(5):373-82.

40 Phillips AC. Blunted cardiovascular reactivity relates to depression, obesity, and self-reported health. Biol Psychol. 2011;86(2):106-13.

41 Carroll D. A brief commentary on cardiovascular reactivity at a crossroads. Biol Psychol. 2011;86(2):149-51.

42 Lovallo D. Do low levels of stress reactivity signal poor states of health? Biol Psychol. 2011;86(2):121-8.

43 Jackson EM, Dishman RK. Cardiorespiratory fitness and laboratory stress: a meta-regression analysis. Psychophysiology. 2006;43(1):57-72. doi:PSYP373.

44 Forcier K, Stroud LR, Papandonatos GD, Hitsman B, Reiches M, Krishnamoorthy J, Niaura R. Links between physical fitness and cardiovascular reactivity and recovery to psychological stressors: a metaanalysis. Health Psychol. 2006;25(6):723-39.

45 Taylor MK, Markham AE, Reis JP, Padilla GA, Potterat EG, Drummond SP, et al. Physical fitness influences stress reactions to extreme military training. Mil Med. 2008;173(8):738-42.

46 Tsutsumi T, Don BM, Zaichkowsky LD, Delizonna LL. Physical fitness and psychological benefits of strength training in community dwelling older adults. Appl Human Sci. 1997;16(6):257-66.
47 Kettunen O, Kyrolainen H, Santtila M, Vasankari T. Physical fitness and volume of leisure time physical activity relate with low stress and high mental resources in young men. J Sports Med Phys Fitness. 2014;54(4):545-51. doi:R40Y2014N04A0545.

48 Childs $\mathrm{E}$, de Wit H. Hormonal, cardiovascular, and subjective responses to acute stress in smokers. Psychopharmacology (Berl). 2009;203(1):1-12. doi:10 1007/s00213-008-1359-5.

49 Hayano J, Yamada M, Sakakibara Y, Fujinami T, Yokoyama K, Watanabe Y, et al. Short- and long-term effects of cigarette smoking on heart rate variability. Am J Cardiol. 1990;65(1):84-8. doi:0002-9149(90)90030-5.

50 Nater UM, Rohleder N. Salivary alpha-amylase as a non-invasive biomarker for the sympathetic nervous system: current state of research.

Psychoneuroendocrinology. 2009:34(4):486-96. doi:10.1016/.j.psyneuen.2009.01.01.

51 Balodis IM, Wynne-Edwards KE, Olmstead MC. The other side of the curve: examining the relationship between pre-stressor physiological responses and stress reactivity. Psychoneuroendocrinology. 2010;35(9):1363-73. doi:10. 1016/j.psyneuen.2010.03.011.

52 Zschucke E, Renneberg B, Dimeo F, Wustenberg T, Strohle A. The stress-buffering effect of acute exercise: evidence for HPA axis negative feedback.

Psychoneuroendocrinology. 2015;51:414-25. doi:10.1016/j.psyneuen.2014.10.019.

53 Nedeljkovic M, Ausfeld-Hafter B, Streitberger K, Seiler R, Wirtz PH. Taiji practice attenuates psychobiological stress reactivity — a randomized controlled trial in healthy subjects. Psychoneuroendocrinology. 2012;37(8): 1171-80. doi:10.1016/j.psyneuen.2011.12.007.

54 Fiocco AJ, Joober R, Lupien SJ. Education modulates cortisol reactivity to the Trier Social Stress Test in middle-aged adults. Psychoneuroendocrinology. 2007:32(8-10):1158-63. doi:S03064530(07)00208-9.

55 Feizi A, Keshteli AH, Nouri F, Roohafza H, Adibi P. A cross-sectional population-based study on the association of personality traits with anxiety and psychological stress: joint modeling of mixed outcomes using shared random effects approach. J Res Med Sci. 2014;19(9):834-43.

56 Fischer JP, Young CN, Fadel PJ. Autonomic adjustments to exercise in humans. Compr Physiol. 2015;5(2):475-512.

57 Longhurst JC, Stebbins CL. The power athlete. Cardiol Clin. 1997;15(3):413-29.

58 Paluska SA, Schwenk TL. Physical activity and mental health: current concepts. Sports Med. 2000;29(3):167-80.

59 Colcombe SJ, Erickson KI, Scalf PE, Kim JS, Rakash R, McAuley E, Elavsky S, Marquez DX, Hu L, Kramer AF. Aerobic exercise training increases brain volume in aging humans. J Gerontol Med Sci. 2006;61(11):1166-70.

60 Erickson Kl, Raji CA, Lopze OL, Becker JT, Rosano C, Newman AB, Gach HM, Thomposn PM, Ho AJ, Kuller LH. Physical activity predicts gray matter volume in late adulthood: The Cardiovascular Health Study. Neurology. 2010:75(16):1415-22

61 Erickson Kl, Voss MW, Prakahs RS, Basak C, Szabo A, Chaddock L,... Kramer F. Exercise training increases size of hippocampus and improves memory. Proc Natl Acad Sci USA. 2011;108(7):3017-22.

62 Gao L, Wang W, Liu D, Zucker $H$. Exercise training normalizes sympathetic outflow by central antioxidant mechanisms in rabbits with pacing-induced chronic heart failure. Circulation. 2007:115(24):3095-102.

63 Bertagnolli M, Schenkel PC, Campos C, Mostarda CT, Casarini DE, Bello-Klein A, Irigoyen $M C$, Rigatto K. Exercise training reduces sympathetic modulation on cardiovascular system and cardiac oxidative stress in spontaneous hypertensive rats. Am J Hypertens. 2008;21(11):1188-93.

64 Hamer H, Sabia S, Batty D, Shipley MJ, Tabak AG, Singh-Manoux A, Kivimaki M. Physical activity and inflammatory markers over 10 years: follow-up in men and women from the Whitehall II Cohort Study. Ciculation. 2012; 126(8):928-33.

65 Thayer JF, Lane RD. Claude Bernard and the heart-brain connection: further elaboration of a model of neurvisceral integration. Neurosci Biobehav Rev. 2009;33(2):81-8. 